\title{
Real-Time Embedded Systems: Present and Future
}

\author{
Christos Koulamas ${ }^{1, *}$ and Mihai T. Lazarescu ${ }^{2}$ (1) \\ 1 Industrial Systems Institute/“Athena” Research Center, PSP Bldg, Stadiou Strt, 26504 Patras, Greece \\ 2 Dipartimento di Elettronica e Telecomunicazioni Politecnico di Torino Turin, I-10129 Turin, Italy; \\ mihai.lazarescu@polito.it \\ * Correspondence: koulamas@isi.gr; Tel.: +30-2610-911-597
}

Received: 10 September 2018; Accepted: 14 September 2018; Published: 18 September 2018

\section{Introduction}

Real-time and networked embedded systems are important bidirectional bridges between the physical and the information worlds. Embedded intelligence increasingly pervades industry, infrastructure, and public and private spaces, being identified as a society and economy emerging "neural system" that supports both societal changes and economic growth. As cost/performance improves, everyday life connected objects increasingly rely on embedded intelligence in an ever-growing array of application fields, specialized technologies, and engineering disciplines.

While this process gradually builds the Internet of Things (IoT), it exposes a series of specific non-trivial timing and other extra-functional requirements and system properties, which are less common in other computing areas. For instance, most embedded systems are cost-sensitive and with real-time constraints, optimized for power and specific tasks, built around a wide array of processors, often resource-constrained, which need to operate under extreme environmental conditions, and where reliability and security can have severe implications.

The area is quite wide, with diverse computer science and engineering fields and practices involved, and the state of the art is mostly captured today in the cyber-physical systems [1] and IoT [2] evolution contexts, addressing design methods and tools [3], operating systems and resource management [4], real-time wireless networking [5], as well as safety and security [6] aspects, either horizontally or vertically along specific application domains.

\section{The Present Issue}

The ten articles in this special issue propose solutions to specific open problems of cyber-physical and real-time embedded systems applicable to both traditional application domains, such as industrial automation and control, energy management, automotive, aerospace and defense systems, as well as emerging domains, such as medical devices, household appliances, mobile multimedia, gaming, and entertainment systems. Specifically, they address important topics related to efficient embedded digital signal processing (DSP), security and safety, scheduling, and support for smart electric grid optimizations.

Efficient digital signal processing is an important enabler for advanced embedded applications in many domains. Of the five articles in this special issue that address efficient embedded DSP and applications, the work in Reference [7] studies feasibility options and evaluates the performance of fully embedded algorithms for real-time ventricular fibrillation detectors which can send timely alerts without requiring external processing, for applications in pervasive health monitoring. Health monitoring applications are also the focus of Reference [8] that proposes an efficient embedded hardware accelerator for long-term bio-signal monitoring and compression, which makes it suitable for various Internet of Things (IoT) applications. Hardware-assisted efficient embedded DSPs are essential in other application domains, too. An FPGA implementation of a multi-band real-time speech 
enhancement system is shown in Reference [9], which includes specific architectural optimizations for speed and energy consumption. An application-specific integrated circuit (ASIC) implementation of real-time and accuracy optimizations for arctangent calculation based on a coordinate rotation digital computer (CORDIC) is shown in Reference [10] (a trigonometric function that is essential for many embedded DSP calculations), while an automated folding scheme for efficient FPGA implementation of fast Fourier transform (FFT), an algorithm widely used in many embedded application domains, is proposed and experimentally evaluated in Reference [11].

As embedded systems pervade most human activities, from households to industry, safety and security architectural and operational aspects become very important. A survey of microarchitectural attacks of embedded systems is presented in Reference [12], which emphasizes potentially very harmful hardware vulnerabilities that usually receive far less attention than the software ones. Middleware often encompasses several embedded and infrastructure systems, which may also be of different types, hence its reliability is highly relevant for the overall security of the applications. In Reference [13], the authors present a formal specification for middleware correctness and a method to thoroughly verify it at runtime. Efficient virtualization for real-time embedded systems is presented in Reference [14], with emphasis on security, safety, functionality and flexible adaptation to most embedded operating systems.

Scheduling for energy efficiency and effective real-time response of embedded systems is addressed in Reference [15], which propose static scheduling methods based on mixed integer linear programming and heuristics, for both periodic and non-periodic tasks.

Finally, as smart electric grids rely (among other requirements) on effective consumer cooperation and coordination to be able to optimize energy production and distribution, the work in Reference [16] proposes several cloud-enabled embedded solutions and provides experimental test and validation results.

\section{Future}

While embedded systems are not novel, they recently accelerated their permeation in most human activities, which increasingly rely on their real-time capability for, e.g., sensing, processing, communication, actuation, and composability.

Their growing importance for the operation and decision-making capability of complex systems raise significant issues regarding many other aspects of their operation. Security, safety, and reliability may directly impact on physical and data safety as embedded systems are increasingly deployed to measure and control human environments, gaining access, and processing sensitive data. Embedded systems control, validation, self-testing, and observability of their programmed or acquired operation are significant concerns for their acceptance in critical infrastructures or operations, while cost, energy, and maintenance requirements become increasingly important to economically sustain the high number of embedded systems expected for IoT applications. Edge processing, on embedded systems, gains importance over data transfer for remote processing to provide a faster real-time response, reduce dependency on data connections, improve scalability, and increase security by reducing the attack surface.

Recent hardware and software [17] advances aimed at efficiently supporting artificial intelligence (IA) applications directly on resource- and energy-constrained embedded systems for a wide class of applications, compound the importance to find effective solutions to above concerns.

Author Contributions: C.K. and M.T.L. worked together during the whole editorial process of the special issue, "Real-Time Embedded Systems", published in the MDPI journal Electronics. M.T.L. drafted this editorial summary. C.K. and M.T.L. reviewed, edited and finalized the manuscript.

Acknowledgments: We thank all authors, who submitted excellent research work to this special issue. We are grateful to all reviewers who contributed evaluations of scientific merits and quality of the manuscripts and provided countless valuable suggestions to improve their quality and the overall value for the scientific community. Our special thanks go to the editorial board of MDPI Electronics journal for the opportunity to guest edit this 
special issue, and to the Electronics Editorial Office staff for the hard and precise work to keep a rigorous peer-review schedule and timely publication.

Conflicts of Interest: The authors declare no conflict of interest.

\section{References}

1. Khaitan, S.K.; McCalley, J.D. Design Techniques and Applications of Cyberphysical Systems: A Survey. IEEE Syst. J. 2015, 9, 350-365. [CrossRef]

2. Samie, F.; Bauer, L.; Henkel, J. IoT technologies for embedded computing: A survey. In Proceedings of the Eleventh IEEE/ACM/IFIP International Conference on Hardware/Software Codesign and System Synthesis (CODES '16), New York, NY, USA, 1-7 October 2016.

3. Jensen, J.C.; Chang, D.H.; Lee, E.A. A model-based design methodology for cyber-physical systems. In Proceedings of the 2011 7th International Wireless Communications and Mobile Computing Conference, Istanbul, Turkey, 4-8 July 2011; pp. 1666-1671.

4. Musaddiq, A.; Zikria, Y.B.; Hahm, O.; Yu, H.; Bashir, A.K.; Kim, S.W. A Survey on Resource Management in IoT Operating Systems. IEEE Access 2018, 6, 8459-8482. [CrossRef]

5. Lu, C.; Saifullah, A.; Li, B.; Sha, M.; Gonzalez, H.; Gunatilaka, D.; Wu, C.; Nie, L.; Chen, Y. Real-Time Wireless Sensor-Actuator Networks for Industrial Cyber-Physical Systems. Proc. IEEE 2016, 104, 1013-1024. [CrossRef]

6. Wolf, M.; Serpanos, D. Safety and Security in Cyber-Physical Systems and Internet-of-Things Systems. Proc. IEEE 2018, 106, 9-20. [CrossRef]

7. Kwon, S.; Kim, J.; Chu, C. Real-Time Ventricular Fibrillation Detection Using an Embedded Microcontroller in a Pervasive Environment. Electronics 2018, 7, 88. [CrossRef]

8. Antonopoulos, C.; Voros, N. A Data Compression Hardware Accelerator Enabling Long-Term Biosignal Monitoring Based on Ultra-Low Power IoT Platforms. Electronics 2017, 6, 54. [CrossRef]

9. Bahoura, M. Pipelined Architecture of Multi-Band Spectral Subtraction Algorithm for Speech Enhancement. Electronics 2017, 6, 73. [CrossRef]

10. Pilato, L.; Fanucci, L.; Saponara, S. Real-Time and High-Accuracy Arctangent Computation Using CORDIC and Fast Magnitude Estimation. Electronics 2017, 6, 22. [CrossRef]

11. Minotta, F.; Jimenez, M.; Rodriguez, D. Automated Scalable Address Generation Patterns for 2-Dimensional Folding Schemes in Radix-2 FFT Implementations. Electronics 2018, 7, 33. [CrossRef]

12. Fournaris, A.; Pocero Fraile, L.; Koufopavlou, O. Exploiting Hardware Vulnerabilities to Attack Embedded System Devices: A Survey of Potent Microarchitectural Attacks. Electronics 2017, 6, 52. [CrossRef]

13. Khan, M.; Serpanos, D.; Shrobe, H. A Formally Reliable Cognitive Middleware for the Security of Industrial Control Systems. Electronics 2017, 6, 58. [CrossRef]

14. Martins, J.; Alves, J.; Cabral, J.; Tavares, A.; Pinto, S. $\mu$ RTZVisor: A Secure and Safe Real-Time Hypervisor. Electronics 2017, 6, 93. [CrossRef]

15. Jiang, X.; Huang, K.; Zhang, X.; Yan, R.; Wang, K.; Xiong, D.; Yan, X. Energy-Efficient Scheduling of Periodic Applications on Safety-Critical Time-Triggered Multiprocessor Systems. Electronics 2018, 7, 98. [CrossRef]

16. Brusco, G.; Burgio, A.; Menniti, D.; Pinnarelli, A.; Sorrentino, N.; Scarcello, L. An Energy Box in a Cloud-Based Architecture for Autonomous Demand Response of Prosumers and Prosumages. Electronics 2017, 6, 98. [CrossRef]

17. Tang, J.; Sun, D.; Liu, S.; Gaudiot, J.L. Enabling Deep Learning on IoT Devices. IEEE Comput. 2017, 50, 92-96. [CrossRef]

(C) 2018 by the authors. Licensee MDPI, Basel, Switzerland. This article is an open access article distributed under the terms and conditions of the Creative Commons Attribution (CC BY) license (http://creativecommons.org/licenses/by/4.0/). 